\title{
Monomial ideals and the failure of the Strong Lefschetz property
}

\author{
Nasrin Altafi ${ }^{1} \cdot$ Samuel Lundqvist ${ }^{2}$
}

Received: 5 January 2021 / Accepted: 7 June 2021 / Published online: 24 June 2021

(c) The Author(s) 2021

\begin{abstract}
We give a sharp lower bound for the Hilbert function in degree $d$ of artinian quotients $\mathbb{k}\left[x_{1}, \ldots, x_{n}\right] / I$ failing the Strong Lefschetz property, where $I$ is a monomial ideal generated in degree $d \geq 2$. We also provide sharp lower bounds for other classes of ideals, and connect our result to the classification of the Hilbert functions forcing the Strong Lefschetz property by Zanello and Zylinski.
\end{abstract}

Keywords Lefschetz properties $\cdot$ Hilbert series $\cdot$ Monomial ideals $\cdot$ Generic forms $\cdot$ Inverse system

Mathematics Subject Classification $13 \mathrm{~A} 02 \cdot 13 \mathrm{D} 40 \cdot 13 \mathrm{E} 10$

\section{Introduction}

An artinian graded algebra has the Weak Lefschetz property (WLP) if there is a linear form $\ell$ such that the map induced by multiplication by $\ell$ is either surjective or injective in every degree, and the element $\ell$ is then called a Weak Lefschetz element, while an artinian graded algebra has the Strong Lefschetz property (SLP) if there is a linear form $\ell$ such that the map induced by multiplication by $\ell^{i}$ is either surjective or injective in every degree, for all $i \geq 1$, and the element $\ell$ is then called a Strong Lefschetz element.

It has long been known that every monomial complete intersection in characteristic zero has the SLP. This result is due to Stanley [15] and independently by Watanabe [19]. One way of thinking about this result is that an artinian algebra of the form $\mathbb{k}\left[x_{1}, \ldots, x_{n}\right] / I$, where $I$ is a monomial ideal, has the SLP when the number of minimal generators is as small as possible (equal to $n$ ). When we increase the number of monomial generators of the ideal, it is not always the case that the quotient algebra has the SLP, or even the WLP. Indeed, for each $n \geq 3$, the equigenerated monomial almost

Nasrin Altafi

nasrinar@kth.se

Samuel Lundqvist

samuel@math.su.se

1 Department of Mathematics, KTH Royal Institute of Technology, Stockholm, Sweden

2 Department of Mathematics, Stockholm University, Stockholm, Sweden 
complete intersection $\mathbb{k}\left[x_{1}, \ldots, x_{n}\right] /\left(x_{1}^{n}, \ldots, x_{n}^{n}, x_{1} \ldots x_{n}\right)$ fails the WLP. This was noticed for $n=3$ by Brenner and Kaid [2], while the proof of the general case $n \geq 4$ is attributed to Migliore et al. [11].

Given these results, it is natural to consider the following question: Suppose that $I$ is an equigenerated artinian monomial ideal such that $\mathbb{k}\left[x_{1}, \ldots, x_{n}\right] / I$ fails one of the Lefschetz properties. What can we say about the number of minimal generators of $I$ ?

The first result in this direction goes back to Mezzetti and Miró-Roig [9] who provided a sharp upper bound for the Hilbert function in degree $d$ of minimal monomial Togliatti systems. Recall that a monomial ideal $I \subset \mathbb{K}\left[x_{1}, \ldots, x_{n}\right]$ generated in degree $d$ is called a monomial Togliatti system if the quotient $\mathbb{k}\left[x_{1}, \ldots, x_{n}\right] / I$ fails the WLP in degree $d-1$ by failing injectivity. Togliatti systems were defined in [10] and the name is in honour of Engenio Togliatti who characterized smooth Togliatti systems for $n=d=3[16,17]$.

The first author and Boij [1] recently gave the following sharp lower bound for the Hilbert function in degree $d$ of an artinian quotient $\mathbb{k}\left[x_{1}, \ldots, x_{n}\right] / I$ failing the WLP, where $I$ is a monomial ideal generated in degree $d$.

Theorem 1 [1, Theorem 1.1, Theorem 1.2] Let $n \geq 3$ and let $\mathbb{k}$ be a field of characteristic zero. Let $I \subset S=\mathbb{k}\left[x_{1}, \ldots, x_{n}\right]$ be a monomial ideal generated in degree $d \geq 2$. Assume that $R=S / I$ fails the WLP. Then

$$
\mathrm{HF}(R, d) \geq \begin{cases}3(d-1) & \text { if } n=3 \text { and } d \text { odd } \\ 3(d-1)+1 & \text { if } n=3 \text { and } d \text { even, } \\ 2 d & \text { if } n \geq 4\end{cases}
$$

where $\mathrm{HF}(R, d)$ is the value of the Hilbert function in degree $d$ of $R$.

Furthermore, the bounds are sharp.

In this note, we carry out a similar program for the SLP, resulting in the following classification.

Theorem 2 Let $n \geq 3$ and let $\mathbb{k}$ be a field of characteristic zero. Let $I \subset S=\mathbb{k}\left[x_{1}, \ldots, x_{n}\right]$ be an artinian monomial ideal generated in degree $d \geq 2$. Let $R=S / I$ and denote the value of the Hilbert function in degree $d$ of $R$ by $\operatorname{HF}(R, d)$.

(i) Assume that $R$ fails the SLP. Then

$$
\mathrm{HF}(R, d) \geq \begin{cases}4 & \text { if } d=2 \\ 3 & \text { if } d \geq 3 .\end{cases}
$$

Moreover, this is sharp in the sense that for any $d$ there is an example for which $\ell^{d-1}: R_{1} \rightarrow R_{d}$ fails to be surjective and the value of the Hilbert function in degree $d$ is given by this bound.

(ii) Let $1 \leq i \leq d-1$. If $\ell^{i}$ fails to have maximal rank in some degree then

$$
\mathrm{HF}(R, d) \geq d-i+2 \text {. }
$$

Moreover, the bound is sharp for every $2 \leq i \leq d-1$ : there is an example where $\ell^{i}: R_{d-i} \rightarrow R_{d}$ is not surjective and the bound is achieved. 
Remark 1 Over a field of characteristic zero, Harima, Migliore, Nagel, and Watanabe showed that every artinian homogeneous quotient in two variables has the SLP [6], explaining why the case $n=2$ is omitted in the results.

Remark 2 In the case $n=3, d=2$, a computation reveals that all quotients of artinian monomial ideals enjoy the WLP and the SLP, and this is the only case for which the conditions in Theorems 1 and 2 are empty.

Remark 3 In Theorem 2 part (ii), the bound holds for every $1 \leq i \leq d-1$ although when $i=1$ multiplication by $\ell$ does not have full rank which means the WLP fails. In this case, the bound in Theorem 2 part (ii) is not sharp.

The consequences of Theorem 2 are stronger than one would first expect, and for $d \geq 3$, we will show that Theorem 2 holds for other natural classes of artinian ideals, including ideals generated by forms of degree $d$. We will see that this generalization gives a bridge to a classification result on the Hilbert functions that force all ideals to have the SLP by Zanello and Zylinski [18].

\section{Preliminaries}

Throughout the remaining part of this paper, let $\mathbb{k}$ be a field of characteristic zero and let $S=\mathbb{k}\left[x_{1}, \ldots, x_{n}\right]$, where $n \geq 3$. We will use that a monomial algebra $R$ has the SLP if and only if $\ell:=x_{1}+\cdots+x_{n}$ is a Strong Lefschetz element [12, Remark 4.5].

Duality arguments will be crucial for our results and we recall here the basic notion of the inverse system. Let $\mathcal{E}=\mathbb{k}\left[y_{1}, \ldots, y_{n}\right]$ be the Macaulay dual ring to $S$, where $S$ acts on $\mathcal{E}$ by differentiation; i.e. $x_{j} \circ f=\partial f / \partial y_{j}$, for every homogeneous polynomial $f \in \mathcal{E}$. There is a bijection between the set of finitely generated $S$-submodules $M$ of $\mathcal{E}$ and the set of artinian quotients $S / I$ given by $I=\operatorname{Ann}_{S}(M)=\left(0:_{S} M\right)$, and $I^{-1}=M$. The module $I^{-1}$ is called the inverse system module of $I$, for more details see [3, 7]. Using this correspondence, we have that $\operatorname{HF}(R, i)=\operatorname{dim}_{\mathbb{k}}\left(I^{-1}\right)_{i}$. For every $i$, the multiplication map $\ell:(S / I)_{i} \rightarrow(S / I)_{i+1}$ has maximal rank if and only if the differentiation map o $\ell:\left(I^{-1}\right)_{i+1} \rightarrow\left(I^{-1}\right)_{i}$ has maximal rank. We observe that when $I$ is a monomial ideal, for every $i$ the $i$ th graded piece of the inverse system module, $\left(I^{-1}\right)_{i}$, is generated by monomials in $\mathcal{E}_{d}$ that are dual to the monomials in $S_{i} \backslash I_{i}$.

Finally, we state one more result from [1] which will be central to our argument.

Theorem 3 [1, Theorem 3.7] Let $f \in \mathcal{E}=\mathbb{k}\left[y_{1}, \ldots, y_{n}\right]$ be a polynomial of degree $d$ such that $\ell^{i} \circ f=0$, for some $1 \leq i \leq d$. Then the number of monomials with non-zero coefficient in $f,|\operatorname{supp}(f)|$, is at least $d-i+2$.

\section{Main result}

Before providing the proof of the main result of the paper we state the following two lemmas in which we provide equivalent conditions for the map $\ell^{i}$ to have maximal rank for $1 \leq i \leq d-1$. Notice that we do not require the ideals in Lemmas 1 and 2 to be equigenerated. 
Lemma 1 Let $R=S / I$ be an artinian algebra, where $n \geq 3$ and $I=\left(m_{1}, \ldots, m_{s}\right)$ a monomial ideal such that $\min \left\{\operatorname{deg}\left(m_{i}\right)\right\}=d \geq 2$. Assume that $\operatorname{HF}(R, d-i) \geq \operatorname{HF}(R, d)$ for some $1 \leq i \leq d-1$. Then the map $\ell^{i}: R_{d-i} \rightarrow R_{d}$ is surjective if and only if the map $\ell^{i}: R_{j} \rightarrow R_{i+j}$ has maximal rank for every $j$.

Proof Suppose that $\ell^{i}: R_{d-i} \rightarrow R_{d}$ is surjective. This means $\left[R / \ell^{i} R\right]_{d}=0$ and also $\left[R / \ell^{i} R\right]_{d+k}=0$ for every $k \geq 1$ which implies that $\ell^{i}: R_{d+k-i} \rightarrow R_{d+k}$ is surjective, for every $k \geq 1$. On the other hand, since degrees of the generators of $I$ is at least $d$, for every $k \geq 1$ the map $\ell^{i}: R_{d-k-i} \rightarrow R_{d-k}$ is trivially injective. The other assertion follows immediately by setting $j=d-i$.

Lemma 2 Let $R=S / I$ be an artinian algebra, where $n \geq 3$ and $I=\left(m_{1}, \ldots, m_{s}\right)$ a monomial ideal such that $\min \left\{\operatorname{deg}\left(m_{i}\right)\right\}=d \geq 2$. Suppose that $\operatorname{HF}(R, 1) \geq \operatorname{HF}(R, d)$. Then $R$ has the SLP if and only if the map $\ell^{d-1}: R_{1} \rightarrow R_{d}$ is surjective.

Proof Assuming that $\operatorname{HF}(R, 1) \geq \operatorname{HF}(R, d)$ implies that $\operatorname{HF}(R, d-i) \geq \operatorname{HF}(R, d)$, for every $1 \leq i \leq d-1$. Now suppose that $\ell^{d-1}: R_{1} \rightarrow R_{d}$ is surjective. Then the map $\ell^{i}: R_{d-i} \rightarrow R_{d}$ is also surjective for every $1 \leq i \leq d-1$. Lemma 1 implies that for every $1 \leq i \leq d-1$ the map $\ell^{i}$ has maximal rank in all degrees. On the other hand, since the map $\ell$ has maximal rank in all degrees, the Hilbert function of $R$ is unimodal, see [6]. Therefore, for every $k \geq 0$ and $1 \leq i \leq d-1$ we have $\operatorname{HF}(R, i) \geq \operatorname{HF}(R, d+k+i)$, so the map $\ell^{d+k}: R_{i} \rightarrow R_{d+k+i}$ has maximal rank if and only if it is surjective. We notice that $\ell^{d+k}: R_{i} \rightarrow R_{d+k+i}$ is the composition of two surjective maps $\ell^{d-i}: R_{i} \rightarrow R_{d}$ and $\ell^{i+k}: R_{d} \rightarrow R_{d+k+i}$ and therefore is surjective. We have shown that $R$ has the SLP. The other implication is trivial.

Now we are able to prove our result for equigenerated monomial ideals failing the SLP.

Proof of Theorem 2 We first prove the second part.

(ii) Fix an integer $i$ such that $1 \leq i \leq d-1$. First assume that $\operatorname{HF}(R, d-i) \geq \operatorname{HF}(R, d)$. Suppose that $\ell^{i}: R_{j} \rightarrow R_{i+j}$ does not have maximal rank for some $j \geq 1$. Using Lemma 1 , we get that the map $\ell^{i}: R_{d-i} \rightarrow R_{d}$ is not surjective. Therefore, Theorem 3 implies that $\operatorname{HF}(R, d) \geq d-i+2$. Now assume that $\operatorname{HF}(R, d)>\operatorname{HF}(R, d-i)$. For $1 \leq i \leq d-1$ we have

$\operatorname{HF}(R, d)>\operatorname{HF}(R, d-i)=\left(\begin{array}{c}n+d-i-1 \\ n-1\end{array}\right) \geq d-i+2$,

where the last inequality follows inductively from

$$
\left(\begin{array}{c}
n+d-i-1 \\
n-1
\end{array}\right)=\frac{n+d-i-1}{n-1}\left(\begin{array}{c}
(n-1)+d-i-1 \\
(n-1)-1
\end{array}\right)
$$

and 


$$
\left(\begin{array}{c}
3+d-i-1 \\
3-1
\end{array}\right)=\left(\begin{array}{c}
d-i+2 \\
2
\end{array}\right) \geq d-i+2 .
$$

In order to show that the bound is sharp for every $2 \leq i \leq d-1$, consider $f=y_{1}^{i-1}\left(y_{2}-y_{3}\right)^{d-i+1} \in \mathcal{E}_{d}$ and let $I \subset S$ to be the monomial ideal generated by monomials in $S_{d}$ dual to the monomials in $\mathcal{E}_{d} \backslash \operatorname{Supp}(f)$. We have that $f \in\left(I^{-1}\right)_{d}$ and

$$
\operatorname{HF}(R, d)=\operatorname{HF}(S, d)-|\mathcal{G}(I)|=|\operatorname{supp}(f)|=d-i+2,
$$

where $\mathcal{G}(I)$ is the minimal generating set of $I$. Observe that $\ell^{i} \circ f=0$ which implies that the differentiation map o $\ell^{i}:\left(I^{-1}\right)_{d} \rightarrow\left(I^{-1}\right)_{d-i}$ is not injective, or equivalently, that $\ell^{i}: R_{d-i} \rightarrow R_{d}$ is not surjective.

(i) Let $d \geq 3$ and assume that $R$ fails the SLP.

First suppose that $\operatorname{HF}(R, 1) \geq \operatorname{HF}(R, d)$. Then using Lemma 2 we conclude that the map $\ell^{d-1}: R_{1} \rightarrow R_{d}$ is not surjective and Theorem 3 implies that $\operatorname{HF}(R, d) \geq 3$. Now suppose we have that $\operatorname{HF}(R, d)>\operatorname{HF}(R, 1)=n \geq 3$, so we get the desired inequality. In this case, $d \geq 3$, the sharpness of the bound is implied by the second part. In fact, the polynomial $f=y_{1}^{d-2}\left(y_{2}-y_{3}\right)^{2}$ is in the kernel of the map $\circ \ell^{d-1}:\left(I^{-1}\right)_{d} \rightarrow\left(I^{-1}\right)_{1}$ and we have that $\operatorname{HF}(R, d)=|\operatorname{supp}(f)|=3$.

Now assume that $d=2$ and that $R$ fails the SLP.

If $n=3$ then a calculation shows that every artinian quadratic monomial ideal in three variables has the SLP, so there is nothing to prove. Thus we assume $n \geq 4$. By Theorem 1, the map $\ell: R_{1} \rightarrow R_{2}$ is surjective when $\operatorname{HF}(R, 2)<4$, so by Lemma $2, R$ has the SLP. Therefore, if $R$ fails the $\operatorname{SLP}$ then $\operatorname{HF}(R, 2) \geq 4$ and the sharpness of the bound is implied by Theorem 1 .

We turn directly to the generalization of Theorem 2 .

Theorem 4 Let $n \geq 3$ and let $\mathbb{k}$ be a field of characteristic zero. Let $I \subset S=\mathbb{k}\left[x_{1}, \ldots, x_{n}\right]$ be an artinian ideal generated in degree $d \geq 3$, or an artinian monomial ideal $\left(m_{1}, \ldots, m_{s}\right)$ such that $\min \left\{\operatorname{deg}\left(m_{i}\right)\right\}=d \geq 3$, or an artinian ideal $\left(f_{1}, \ldots, f_{s}\right)$ such that $\min \left\{\operatorname{deg}\left(f_{i}\right)\right\}=d \geq 3$. Let $R=S /$ I and denote the value of the Hilbert function in degree $d$ of $R$ by $\operatorname{HF}(R, d)$.

(i) Assume that $R$ fails the SLP. Then

$$
\mathrm{HF}(R, d) \geq 3 \text {. }
$$

Moreover, this is sharp in the sense that for any $d$ there is an example belonging to the specific class for which $\ell^{d-1}: R_{1} \rightarrow R_{d}$ fails to be surjective.

(ii) Let $1 \leq i \leq d-1$. If $\ell^{i}$ fails to have maximal rank in some degree then

$$
\mathrm{HF}(R, d) \geq d-i+2 \text {. }
$$

Moreover, the bound is sharp for every $2 \leq i \leq d-1$ : there is an example where $\ell^{i}: R_{d-i} \rightarrow R_{d}$ is not surjective and the bound is achieved.

Proof Let $R^{\prime}=S / J$ where $J=$ in $(I)$ is the initial ideal of $I$ with respect to a term order. Notice that $J$ is a monomial ideal such that $\min \left\{\operatorname{deg}\left(m_{i}\right)\right\}=d \geq 3$ and therefore Lemmas 1 and 2 hold for $J$. 
Wiebe's result [20, Proposition 2.9] states that if $R^{\prime}$ has the SLP then the same holds for $R$. The proof of [20, Proposition 2.9] actually reveals a more general fact: for a general enough $\tilde{\ell}$ and for every $i, j \geq 0, \tilde{\ell}^{j}: R_{i} \rightarrow R_{i+j}$ has maximal rank if $\ell^{j}: R_{i}^{\prime} \rightarrow R_{i+j}^{\prime}$ has maximal rank.

The inequalities now follow by the same line as of the proof of Theorem 2. For the sharpness parts, we use that artinian monomial ideals generated in degree $d$ are part of each of the three classes.

Remark 4 For $d=2$, the bounds in Theorems 1 and 2 coincide, and in fact, in the proof of Theorem 2, we use Theorem 1 to obtain sharp bounds for $\operatorname{HF}(R, 2)$ for artinian monomial ideals generated in degree 2. But for the classes of artinian ideals in Theorem 4, the same approach does not provide a bound for $\operatorname{HF}(R, 2)$, since Theorem 1 is only valid for equigenerated monomial ideals.

However, when $n=3$, if a quadratic artinian ideal $I$ fails the $\operatorname{SLP}$, then $\operatorname{HF}(R, 2) \geq 4$, which coincides with the bound given in Theorem 2. To see this, notice that if $\operatorname{HF}(R, 2) \leq 2$, then Theorem 3 implies that $\ell:[S / \text { in }(I)]_{1} \rightarrow[S / \text { in }(I)]_{2}$ is surjective and therefore, using Lemma 2, we have that $S /$ in $(I)$, and thus $R$, has the $\operatorname{SLP}$. If $\operatorname{HF}(R, 2)=3$, then $I$ is a complete intersection having Hilbert function $(1,3,3,1)$, which has the SLP according to the Gordan-Noether theorem [4].

\section{A connection to the Migliore-Zanello and the Zanello-Zylinski classification results}

Migliore and Zanello [13] classified the Hilbert functions that force the WLP, and later Zanello and Zylinski [18] classified the Hilbert functions that force the SLP.

Theorem 5 Let $\mathrm{H}: 1, h_{1}=n, h_{2}, \ldots, h_{e}, h_{e+1}=0$, be a possible Hilbert function, according to Macaulay's theorem [8], and let $t$ be the smallest integer such that $h_{t} \leq t$. Then we have the following.

(i) [13, Theorem 5] All artinian algebras having the Hilbert function $\mathrm{H}$ enjoy the WLP if and only if, for all $i=1,2, \ldots, t-1$, we have

$$
h_{i-1}=\left(\left(h_{i}\right)_{(i)}\right)_{-1}^{-1} .
$$

(ii) $[18$, Theorem 3.2] All artinian algebras having the Hilbert function $\mathrm{H}$ enjoy the SLP if and only if

- $n=2 ;$ or

- $n>2, h_{t} \leq 2$, and, for all $i=1,2, \ldots, t-1$, we have

$$
h_{i-1}=\left(\left(h_{i}\right)_{(i)}\right)_{-1}^{-1} \text {. }
$$


In the theorem above, for integers $m$ and $i$, the $i$-binomial expansion of $m$ is denoted by $m_{(i)}$, that is,

$$
m=m_{(i)}=\left(\begin{array}{c}
m_{i} \\
i
\end{array}\right)+\left(\begin{array}{c}
m_{i-1} \\
i-1
\end{array}\right)+\cdots+\left(\begin{array}{c}
m_{j} \\
j
\end{array}\right),
$$

where $m_{i} \geq m_{i-1} \geq \cdots \geq j \geq 1$. Also

$$
\left(m_{(i)}\right)_{-1}^{-1}=\left(\begin{array}{c}
m_{i}-1 \\
i-1
\end{array}\right)+\left(\begin{array}{c}
m_{i-1}-1 \\
i-1-1
\end{array}\right)+\cdots+\left(\begin{array}{c}
m_{j}-1 \\
j-1
\end{array}\right),
$$

where $\left(\begin{array}{l}c \\ d\end{array}\right)=0$ whenever $c<d$ or $d<0$.

We will now discuss how Theorem 5 is related to the results in Theorems 1, 2, and 4 .

We consider first the WLP and the case $n \geq 4$. Suppose $I$ is a monomial ideal equigenerated in degree $d$ and $\operatorname{HF}(R, d) \leq d$. Both Theorems 1 and 5 part (i) imply that $R$ has the WLP. Now let $\operatorname{HF}(R, d)=i$ and suppose that $d+1 \leq i \leq 2 d-1$. Theorem 1 implies that $R$ has the WLP. On the other hand,

$$
i_{(d)}=\left(\begin{array}{c}
d+1 \\
d
\end{array}\right)+\left(\begin{array}{l}
d-1 \\
d-1
\end{array}\right)+\cdots+\left(\begin{array}{c}
d-(i-(d+1)) \\
d-(i-(d+1))
\end{array}\right),
$$

so $\left(i_{(d)}\right)_{-1}^{-1}=i-1 \leq 2 d-2 \neq \operatorname{HF}(R, d-1)$. Therefore, the requirement of Theorem 5 part (i) is not satisfied. This shows that there is an artinian ideal such that $\operatorname{HF}(R, d)=i$ which fails the WLP. Moreover, by considering the initial ideal of $I$, Wiebe's result implies that there is an artinian monomial ideal such that $\operatorname{HF}(R, d)=i$ which fails the WLP. A similar argument can be applied for the $n=3$ case.

We now turn to the SLP. If $I$ is generated in degrees $d \geq 2$ and $\operatorname{HF}(R, d) \leq 2$ then one can easily check that $t=d$ in Theorem 5 part (ii), so $R$ has the SLP. This gives an alternative proof of one direction in part (i) of Theorem 4.

In the quadratic case however, for each $n \geq 3$ there is an artinian ideal such that $\mathrm{HF}(R, 2)=3$ and for which the SLP fails for $R$ by Theorem 5 part (ii), although for all monomial artinian equigenerated ideals such that $\operatorname{HF}(R, d)=3$, the SLP holds according to Theorem 2. Moreover, by considering the initial ideal, by Wiebe's result we conclude that there is an artinian monomial ideal such that $\operatorname{HF}(R, d)=3$ which fails the SLP.

Acknowledgements The initial investigation was performed with the help of Macaulay2 [5] and the package Maximal rank properties [14]. The authors thank the anonymous referee for useful comments that improved both the results and the presentation of the paper.

Funding Open access funding provided by Stockholm University.

Open Access This article is licensed under a Creative Commons Attribution 4.0 International License, which permits use, sharing, adaptation, distribution and reproduction in any medium or format, as long as you give appropriate credit to the original author(s) and the source, provide a link to the Creative Commons licence, and indicate if changes were made. The images or other third party material in this article are included in the article's Creative Commons licence, unless indicated otherwise in a credit line to the material. If material is not included in the article's Creative Commons licence and your intended use is not permitted by statutory regulation or exceeds the permitted use, you will need to obtain permission directly from the copyright holder. To view a copy of this licence, visit http://creativecommons.org/licenses/by/4.0/. 


\section{References}

1. Altafi, N., Boij, M.: The weak Lefschetz property of equigenerated monomial ideals. J. Algebra 556, 136-168 (2020)

2. Brenner, H., Kaid, A.: A note on the weak Lefschetz property of monomial complete intersections in positive characteristic. Collect. Math. 62(1), 85-93 (2011)

3. Geramita, A.V.: Inverse systems of fat points: Waring's problem, secant varieties of Veronese varieties and parameter spaces for Gorenstein ideals. In: The Curves Seminar at Queen's, vol. 10, pp. 2-114 (1996)

4. Gordan, P., Nöther, M.: Ueber die algebraischen formen, deren hesse'sche determinante identisch verschwindet. Math. Ann. 4(10), 547-568 (1876)

5. Grayson, D., Stillman, M.: Macaulay2, a software system for research in algebraic geometry. http:// www2.macaulay2.com/Macaulay2/Citing/. Accessed June 2021

6. Harima, T., Migliore, J., Nagel, U., Watanabe, J.: The weak and strong Lefschetz properties for Artinian K-algebras. J. Algebra 262, 99-126 (2003)

7. Iarrobino, A., Kanev, V.: Power Sums, Gorenstein Algebras, and Determinantal Loci. Appendix C by Iarrobino and Steven L. Kleiman. Lecture Notes in Mathematics, vol. 1721. Springer, Berlin (1999)

8. Macaulay, F.S.: Some properties of enumeration in the theory of modular systems. Proc. Lond. Math. Soc. 2(26), 531-555 (1927)

9. Mezzetti, E., Miró-Roig, R.M.: The minimal number of generators of a Togliatti system. Ann. Mat. Pura Appl. 195, 2077-2098 (2016)

10. Mezzetti, E., Miró-Roig, R.M., Ottaviani, G.: Laplace equations and the weak Lefschetz property. Can. J. Math. 65(3), 634-654 (2013)

11. Migliore, J., Miró-Roig, R.M., Nagel, U.: Monomial ideals, almost complete intersections and the weak Lefschetz property. Trans. Am. Math. Soc. 363, 229-257 (2011)

12. Migliore, J., Nagel, U.: A tour of the weak and strong Lefschetz properties. J. Commut. Algebra 5(3), 329-358 (2013)

13. Migliore, J., Zanello, F.: The Hilbert functions which force the Weak Lefschetz property. J. Pure Appl. Algebra 210(2), 465-471 (2007)

14. Nicklasson, L.: Maximal rank properties, a Macaulay2 package (2019). https://github.com/LisaNickla sson/MaximalRankProperties-Macaulay2-package. Accessed 1 June 2021

15. Stanley, R.P.: Weyl groups, the hard Lefschetz theorem, and the Sperner property. SIAM J. Algebraic Discrete Methods 1(2), 168-184 (1980)

16. Togliatti, E.: Alcuni esempî di superficie algebriche degli iperspazî che rappresentano un' equazione di Laplace. Commun. Math. Helv. 1, 255-272 (1929)

17. Togliatti, E.: Alcune osservazioni sulle superficie razionali che rappresentano equazioni di Laplace. Ann. Mat. Pura Appl. 25, 325-339 (1946)

18. Zanello, F., Zylinski, J.V.: Forcing the strong Lefschetz and the maximal rank properties. J. Pure Appl. Algebra 213(6), 1026-1030 (2009)

19. Watanabe, J.: The Dilworth number of Artinian rings and finite posets with rank function. In: Commutative Algebra and Combinatorics, Advanced Studies in Pure Math. 11, pp. 303-312. Kinokuniya Co., North Holland, Amsterdam (1987)

20. Wiebe, A.: The Lefschetz property for component wise linear ideals and Gotzmann ideals. Commun. Algebra 32(12), 4601-4611 (2004)

Publisher's Note Springer Nature remains neutral with regard to jurisdictional claims in published maps and institutional affiliations. 